\title{
TECNOLOGIA BOTA DE UNNA NA CICATRIZAÇÃO DA ÚLCERA VARICOSA*
}

\author{
Mitzy Tannia Reichembach Danski ${ }^{1}$, Deise Cristina Furtado Liedke ${ }^{2}$, Stela Adami Vayego ${ }^{3}$, Letícia Pontes ${ }^{4}$, \\ Jolline Lind ${ }^{5}$, Derdried Athanasio Johann ${ }^{6}$
}

\begin{abstract}
RESUMO: Objetivou-se analisar a eficiência da tecnologia Bota de Unna no processo de cicatrização de úlceras varicosas. Trata-se de pesquisa descritiva retrospectiva, realizada no ambulatório de tratamento de feridas de um hospital de ensino da região sul do Brasil, tendo como fonte de dados prontuários de pacientes portadores de úlcera venosa e que utilizaram a Bota de Unna. Incluiu-se 49 pacientes que apresentaram 80 úlceras varicosas, predominantemente em membro inferior direito com $56,2 \%(n=45)$ e região de maléolo medial com $42,5 \%$ $(\mathrm{n}=34)$. Em relação à cicatrização das úlceras, houve significância estatística para volume intenso do exsudato ( $p$-valor $=0,0193)$ e tamanho da úlcera $(p$-valor $<0,0001)$, tendência para deambulação sem auxílio $(p$-valor $=0,0986)$ e exsudato purulento ( $p$-valor=0,0629) como variáveis que interferem na cicatrização. Quarenta e um pacientes $(51,3 \%)$ tiveram cicatrização das lesões mediante o uso da Bota de Unna em até 12 semanas. A tecnologia foi eficiente na cicatrização das úlceras varicosas, em especial nas úlceras de pequeno tamanho.

DESCRITORES: Úlcera varicosa; Tecnologia; Cicatrização; Pesquisa em enfermagem clínica; Cuidados de enfermagem.

\section{UNNA BOOT TECHNOLOGY IN THE HEALING OF VARICOSE ULCERS}

ABSTRACT: This study aimed to analyze the efficiency of Unna Boot technology in the varicose ulcer healing process. This was a retrospective descriptive study conducted in the wound care clinic of a teaching hospital in southern Brazil, using as the data source the medical records of patients with venous ulcers that used the Unna Boot. The study included 49 patients with 80 varicose ulcers, predominantly in the right lower limb, with $56.2 \%(n=45)$, and the medial malleolus region, with $42.5 \%(n=34)$. Regarding the healing of the ulcers, a large volume of exudate $(p=0.0193)$ and ulcer size $(p<0.0001)$ were statistically significant, while walking without assistance $(p=0.0986)$ and purulent exudate $(p=0.0629)$ showed a tendency, as variables that affected the healing. Forty-one patients $(51.3 \%)$ presented wound healing with the use of the Unna Boot within 12 weeks. The technology is effective in healing varicose ulcers, especially smaller ulcers.

DESCRIPTORS: Varicose ulcer; Technology; Healing; Research in clinical nursing; Nursing care.
\end{abstract}

\section{TECNOLOGÍA BOTA DE UNNA EN LA CICATRIZACIÓN DE ÚLCERA VARICOSA}

RESUMEN: Estudio cuyo objetivo fue analizar la eficiencia de la tecnología Bota de Unna en el proceso de cicatrización de úlceras varicosas. Fue una investigación descriptiva retrospectiva, realizada en el ambulatorio de tratamiento de heridas de un hospital de enseñanza de la región sur de Brasil, que tuvo como fuente de datos prontuarios de pacientes con úlcera venosa que utilizaron la Bota de Unna. Participaron 49 pacientes que presentaron 80 úlceras varicosas, con predominancia en el miembro inferior derecho con $56,2 \%$ ( $n=45)$ y región de maléolo medial con $42,5 \%(n=34)$. Acerca de la cicatrización de las úlceras, hubo significancia estadística para volumen intenso de exudato( $p$-valor $=0,0193$ ) y tamaño de la úlcera ( $p$-valor $<0,0001$ ), tendencia para deambulación sin ayuda ( $p$-valor $=0,0986$ ) y exudato purulento ( $p$-valor $=0,0629$ ) como variables que interfieren en la cicatrización. Cuarenta y uno pacientes $(51,3 \%)$ presentaron cicatrización de las lesiones a causa del uso de la Bota de Unna en hasta 12 semanas. La tecnología fue eficiente en la cicatrización de las úlceras varicosas, especialmente en las de pequeño tamaño.

DESCRIPTORES: Úlcera varicosa; Tecnología; Cicatrizacióno; Investigación en enfermería clínica; Cuidados de enfermería.

*Artigo extraído da dissertação de mestrado intitulada: "Uso da bota de unna como tecnologia no cuidado de Enfermagem em Úlcera Venosa". Universidade Federal do Paraná, 2014.

${ }^{1}$ Enfermeira. Doutora em História. Docente de Enfermagem e do Programa de Pós-Graduação em Enfermagem da Universidade Federal do Paraná. Curitiba, PR, Brasil.

${ }^{2}$ Enfermeira. Mestre em Enfermagem. Hospital de Clínicas da Universidade Federal do Paraná. Curitiba, PR, Brasil.

${ }^{3}$ Matemática. Doutora em Genética. Docente da Universidade Federal do Paraná. Curitiba, PR, Brasil.

${ }^{4}$ Enfermeira. Doutora em Educação. Docente de Enfermagem da Universidade Federal do Paraná. Curitiba, PR, Brasil.

${ }^{5}$ Enfermeira. Mestranda em Enfermagem. Universidade Federal do Paraná. Curitiba, PR, Brasil.

${ }^{6}$ Enfermeira. Doutora em Enfermagem. Instituto Federal do Paraná. Curitiba, PR, Brasil.

Autor Correspondente:

Recebido: 12/02/2016

Jolline Lind

Finalizado: 12/09/2016

Universidade Federal do Paraná

Av. Lothario Meissner, 632 - 80210-170 - Curitiba, PR, Brasil

E-mail: lind.jolline@gmail.com 


\section{INTRODUÇÃO}

As úlceras varicosas definem-se por lesões que se desenvolvem em membros inferiores relacionadas à insuficiência venosa ${ }^{(1)}$. Constituem importante problema de saúde pública pela elevada incidência, prevalência, cronicidade, impacto socioeconômico e frequência de recidivas. No tratamento da úlcera varicosa o enfermeiro exerce papel fundamental, tendo em vista a responsabilidade do cuidado direto $^{(2)}$, incluindo o acompanhamento contínuo do processo de cicatrização.

As tecnologias para o cuidado, parte do processo de enfermagem, permitem ao enfermeiro oferecer o melhor tratamento clínico ao paciente com úlcera varicosa. Essas tecnologias incluem a realização do curativo, terapia compressiva, orientações quanto à importância do repouso e uso de meias de compressão após a cicatrização(3).

A terapia compressiva apresenta-se como importante alternativa de tratamento para pacientes com úlcera varicosa. Esta modalidade de tratamento está disponível de duas formas: a terapia elástica ou bandagem de longo estiramento, que consiste em um sistema de multicamadas, e terapia inelástica ou bandagem de curto estiramento, conhecida como Bota de Unna, objeto dessa pesquisa ${ }^{(4)}$.

A Bota de Unna, desenvolvida por um dermatologista alemão em 1896, caracteriza-se por bandagem compressiva composta por óxido de zinco, gelatina, calamina e glicerina ${ }^{(5)}$. É possível a permanência por até sete dias associada a outra tecnologia como a gaze não aderente, espumas, prata nanocristalina e hidrofibra com prata ${ }^{(6)}$.

O interesse na investigação sobre o tema se deu pela percepção empírica, por um período de 13 anos, da pesquisadora no tratamento de pacientes com úlceras varicosas. Durante este período, observou-se que os pacientes tratados com a Bota de Unna apresentaram melhor evolução clínica em comparação àqueles submetidos a outros tratamentos.

Considerando que a aplicação de uma tecnologia em saúde, direcionada a um determinado problema em condições reais de uso, pode beneficiar indivíduos de uma população definida ${ }^{(7)}$, este estudo teve como objetivo analisar a eficiência da tecnologia Bota de Unna no processo de cicatrização da úlcera varicosa.

\section{MÉTODO}

Pesquisa descritiva retrospectiva realizada no ambulatório de tratamento de feridas de um hospital de ensino da região Sul do Brasil. Para seu desenvolvimento, foram analisados os prontuários de pacientes com diagnóstico de úlcera varicosa, tratados com a tecnologia Bota de Unna, entre janeiro de 2007 e dezembro de 2012, perfazendo um período de cinco anos.

Neste ambulatório atuam duas enfermeiras, no período da manhã e tarde, as quais realizam o processo de enfermagem mediante a consulta de enfermagem, avaliação das lesões, implantação e continuidade do tratamento, orientações ao paciente e familiar e/ou cuidador de acordo com protocolo institucional. As úlceras varicosas foram acompanhadas pelas duas profissionais supracitadas durante todo o período de tratamento.

A obtenção dos dados ocorreu nos meses de março e abril de 2014, por meio de instrumentos previamente testados. Na primeira etapa da pesquisa, empregou-se instrumento com a finalidade caracterizar o perfil epidemiológico dos pacientes com úlceras crônicas, com o objetivo de identificar os pacientes portadores de úlceras varicosas. Na sequência, analisaram-se as variáveis sociodemográficas e clínicas dos pacientes e a avaliação da úlcera.

Os critérios de inclusão abrangeram prontuários disponíveis de todos os pacientes em atendimento no Ambulatório de Tratamento de Feridas, com diagnóstico de úlcera varicosa, tratados com a tecnologia Bota de Unna, no período de janeiro de 2007 a dezembro de 2012. Excluíram-se os prontuários não disponíveis para a coleta de dados.

Considerou-se para esta pesquisa, como eficiência da tecnologia Bota de Unna, a cicatrização da 
úlcera varicosa. A úlcera foi avaliada como cicatrizada quando se apresentou totalmente epitelizada, sem solução de continuidade da pele. As medidas das úlceras varicosas foram realizadas por meio de mensuração linear com o papel de acetato, colocado em contato direto com o leito da ferida e o desenho transcrito através da técnica de decalque, para o prontuário do paciente. A área de superfície estimada em centímetros quadrados foi obtida multiplicando-se as duas medidas (comprimento $\mathrm{x}$ largura), lembrando que o comprimento refere-se à medida no sentido vertical ou céfalo-caudal e a largura refere-se à medida horizontal.

Para a análise dos resultados, os dados foram digitados em planilhas eletrônicas do programa Microsoft Excel $^{\circledast}$ e com auxílio do programa Bioestat ${ }^{\circledast}$ analisados mediante estatística descritiva. Na análise de fatores que possam interferir no desfecho cicatrização, foram utilizados o teste do Quiquadrado, teste Exato de Fisher e teste G de Willians para variáveis categóricas e utilizou-se nível de significância de $5 \%$.

O projeto foi aprovado pelo comitê de ética da Instituição, conforme parecer número 411.492, sendo respeitado os preceitos éticos previstos na Resolução 466/2012, que estabelece as diretrizes para pesquisas envolvendo seres humanos ${ }^{(8)}$.

\section{RESULTADOS}

Foram analisados 142 prontuários, totalizando 252 úlceras crônicas, com predomínio de úlceras varicosas, $n=112(44,4 \%)$, seguidas das provenientes de hanseníase $n=32(12,6 \%)$. Foram identificados 49 pacientes que foram tratados com a Bota de Unna e 80 lesões, pois alguns pacientes apresentavam mais de uma úlcera varicosa. Predominou na amostra pacientes do sexo masculino $57,1 \%$ ( $\mathrm{n}=28$ ) com média de 57 anos de idade, mínimo de 25 e máximo de 84 anos. Entre os participantes, 61,2\% ( $n=30)$ eram casados e $81,6 \%(n=40)$ de etnia caucasoide. Em relação à procedência, $53,1 \%(n=26)$ eram de Curitiba e os demais da região metropolitana e municípios do interior do Estado do Paraná. Quanto à escolaridade, 69,3\% ( $n=34)$ concluíram o ensino fundamental, 24,2 \% $(n=12)$ o ensino médio e três $(6,1 \%)$ não eram alfabetizados. Em relação a comorbidades, $51 \%(n=25)$ apresentavam hipertensão arterial sistêmica, $77,6 \%(n=38)$ diabetes, as duas comorbidades associadas $73,47 \%(n=36)$ e $93,9 \%$ $(\mathrm{n}=46)$ negaram tabagismo.

Dos 49 pacientes pesquisados, $61,2 \%(n=30)$ apresentaram uma úlcera, nove $(18,4 \%)$ duas úlceras, nove $(18,4 \%)$ três úlceras e um $(2 \%)$ cinco úlceras, totalizando 80 úlceras varicosas. Quanto à localização e região, 56,2\% $(n=45)$ das úlceras localizavam-se em membro inferior direito e predominantemente em região de maléolo medial $42,5 \%(n=34)$. Quanto ao tempo em que o paciente foi acometido pela úlcera varicosa, a média foi de 1355 dias, com intervalo de tempo entre 30 dias e 10220 dias (28 anos), isto devido aos fatores relacionados ao encaminhamento deste paciente ao serviço, pois muitos quando chegaram ao ambulatório já apresentavam as úlceras há muitos anos com ou sem tratamento em outro serviço.

$\mathrm{Na}$ avaliação do leito da úlcera varicosa, as características predominantes nas lesões foram presença de esfacelos em $57,5 \%(n=46)$, exsudato de aspecto seroso em $78,7 \%(n=63)$, volume moderado de exsudato em $53,7 \%(n=43)$ e odor discreto em $80 \%(n=64)$. Observou-se dor moderada registrada em $33,7 \%(n=27)$, seguida de dor leve em $30 \%(n=25)$ e dor intensa em $23,7 \%(n=19)$. Dentre as coberturas associadas à Bota de Unna, as mais utilizadas foram prata nanocristalina $37,5 \%(n=30)$ e curativo de carvão ativado com prata 32,5\% (n=26). Outras coberturas utilizadas com menor frequência incluíram a hidrocelular em $15 \%(n=12)$, hidrofibra com prata em seis $(7,5 \%)$, silicone com prata em quatro $(5 \%)$, colágeno/alginato em duas $(1,2 \%)$.

Com relação ao tamanho das úlceras, foram estabelecidos nesta pesquisa para fins de análise os seguintes critérios: úlcera varicosa pequena de 0,1 a $10 \mathrm{~cm} 2$; média de 10,1 a $100 \mathrm{~cm} 2$ e grande a partir de $100,1 \mathrm{~cm} 2$. Houve predomínio de lesões pequenas $55 \%(\mathrm{n}=44)$, seguida de lesões médias $23,7 \%$ $(n=19)$ e grandes $21,3 \%(n=17)$.

Quanto às variáveis relacionadas ao paciente, apenas a deambulação apresentou tendência para retardar a cicatrização da úlcera varicosa em até 12 semanas, demonstrada na Tabela 1. 
Quanto às características clínicas da úlcera varicosa, as variáveis exsudato de volume intenso e odor ausente apresentaram significância estatística e exsudato purulento tendência para interferência na cicatrização, observado na Tabela 2.

Tabela 1 - Distribuição da frequência e percentual das variáveis dos pacientes com úlcera varicosa de acordo com o tempo de cicatrização. Curitiba, PR, Brasil, 2014

\begin{tabular}{|c|c|c|c|c|c|c|}
\hline \multirow[t]{2}{*}{ Variáveis } & \multicolumn{5}{|c|}{ Cicatrização até 12 semanas $(n=49)$} & \multirow[b]{2}{*}{ p-valor } \\
\hline & Não & $\%$ & Sim & $\%$ & Total & \\
\hline \multicolumn{7}{|l|}{ Hipertensão } \\
\hline Não & 15 & 53,5 & 9 & 42,8 & 24 & $0,3254^{*}$ \\
\hline Sim & 13 & 46,4 & 12 & 57,1 & 25 & \\
\hline \multicolumn{7}{|l|}{ Diabetes } \\
\hline Não & 23 & 82,1 & 15 & 71,4 & 38 & $0,2918^{*}$ \\
\hline Sim & 5 & 17,8 & 6 & 28,5 & 11 & \\
\hline \multicolumn{7}{|l|}{ Tabagismo } \\
\hline Não & 27 & 96,4 & 19 & 90,4 & 46 & $0,3913^{*}$ \\
\hline Sim & 1 & 3,5 & 2 & 9,5 & 3 & \\
\hline \multicolumn{7}{|l|}{ Mobilidade } \\
\hline Deambula & 27 & 96,4 & 17 & 80,9 & 44 & $0,0986^{*}$ \\
\hline Deambula com auxílio & 1 & 3,5 & 4 & 19 & 5 & \\
\hline Total & 28 & 100 & 21 & 100 & 49 & \\
\hline
\end{tabular}

Legenda: * Teste de Fisher

Tabela 2 - Distribuição da frequência e percentual das variáveis das úlceras varicosas de acordo com o tempo de cicatrização. Curitiba, PR, Brasil, 2014

\begin{tabular}{|c|c|c|c|c|c|}
\hline \multirow[t]{2}{*}{ Variáveis } & \multicolumn{5}{|c|}{ Cicatrização em até 12 semanas $(n=80)$} \\
\hline & Sim & $\%$ & Não & $\%$ & p-valor \\
\hline \multicolumn{6}{|l|}{ Exsudato } \\
\hline Purulento & 0 & 0 & 3 & 7,5 & $0,0629 * *$ \\
\hline Sero-sanguinolento & 5 & 12,5 & 9 & 22,5 & \\
\hline Seroso & 35 & 87,5 & 28 & 70 & \\
\hline \multicolumn{6}{|l|}{ Volume do Exsudato } \\
\hline Intenso & 5 & 12,5 & 16 & 40 & $0,0193+$ \\
\hline Moderado & 26 & 65 & 17 & 42,5 & \\
\hline Pouco & 9 & 22,5 & 7 & 17,5 & \\
\hline \multicolumn{6}{|l|}{ Dor } \\
\hline Ausente & 6 & 15 & 3 & 7,5 & $0,274^{* *}$ \\
\hline Intensa & 6 & 15 & 13 & 32,5 & \\
\hline Leve & 13 & 32,5 & 12 & 30 & \\
\hline Moderada & 15 & 37,5 & 12 & 30 & \\
\hline \multicolumn{6}{|l|}{ Odor } \\
\hline Ausente & 7 & 17,5 & 2 & 5 & $0,039^{* *}$ \\
\hline Discreto & 32 & 80 & 32 & 80 & \\
\hline Intenso & 1 & 2,5 & 6 & 15 & \\
\hline
\end{tabular}

Legenda: ** Teste G de Willians; † Teste Qui-quadrado 
Não houve diferença estatística entre a porcentagem de úlceras cicatrizadas até 12 semanas e após 12 semanas. Entretanto, quando discriminada pelo tamanho da úlcera, observa-se maior eficiência da tecnologia Bota de Unna quando se tratam de úlceras pequenas: 15\% $(n=12)$ cicatrizaram após 12 semanas contra $40 \%(n=32)$ de cicatrização até 12 semanas, ( $p$-valor $<0,0001)$. Para as úlceras médias, $16,3 \%(n=13)$ cicatrizaram após 12 semanas e seis $(7,5 \%)$ em até 12 semanas, e para as úlceras grandes $17,5 \%(n=14)$ cicatrizaram após 12 semanas e apenas três (3,8\%) tiveram cicatrização em até 12 semanas, conforme Figura 1.

Considerando-se todas as úlceras analisadas, evidenciou-se correlação positiva e significativa $(r=$ $60,08 \%$, p-valor $<0,0001$ ) entre a área inicial da ferida e o tempo para cicatrização, indicando que quanto maior a área da ferida maior o tempo de cicatrização, conforme Figura 2.

Observou-se que a área das úlceras varicosas variou de 0,24cm2 a $237 \mathrm{~cm} 2$, com média de 38,71 $\mathrm{cm} 2$. Após quatro semanas de tratamento, a área média das lesões era de 19,76 cm2 e ao final de 12 semanas a área média era de 5,63 cm2. Observou-se significativa redução da área da lesão ao final de 12 semanas, demonstrando que a tecnologia Bota de Unna é eficiente no processo de cicatrização de úlceras varicosas ( $p$-valor $<0,0001)$.

Outro dado importante encontrado neste estudo é que todos os pacientes que utilizaram a Bota de Unna por 3 a 4 semanas, após a cicatrização da úlcera como forma de prevenção, não apresentaram recidivas.

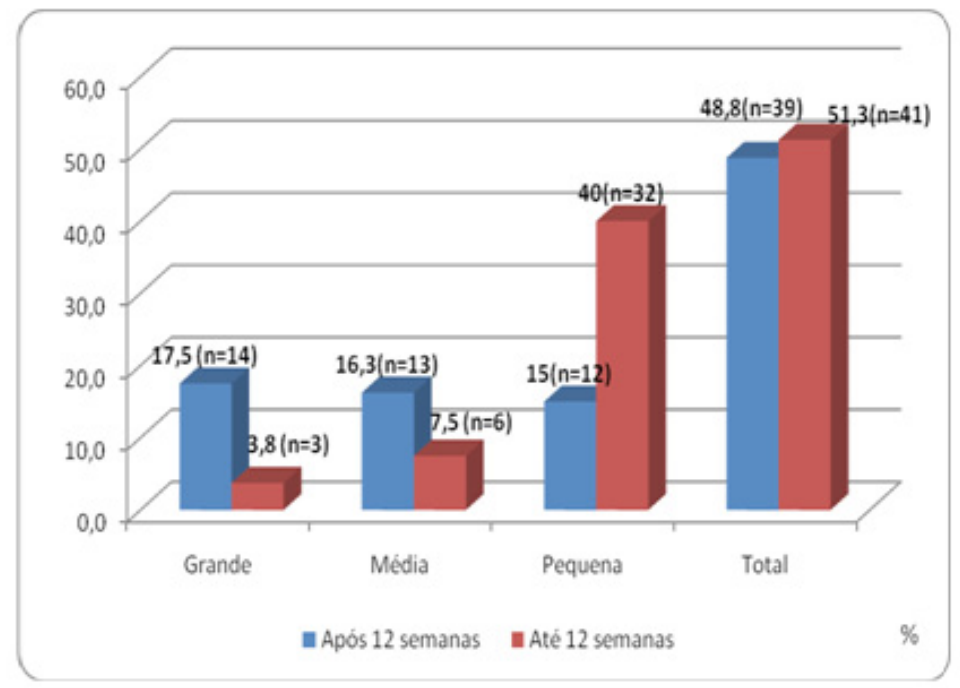

Figura 1 - Distribuição percentual e numérica da cicatrização com uso de Bota de Unna em relação ao tamanho da úlcera varicosa. Curitiba, PR, Brasil, 2014

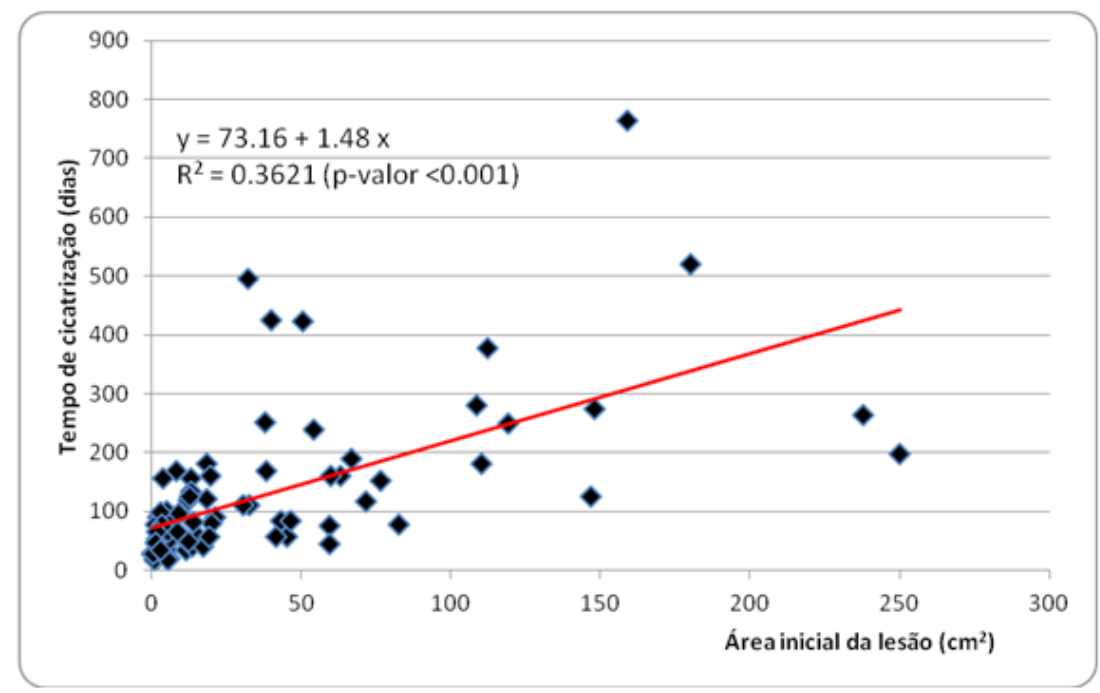

Figura 2 - Correlação entre área inicial da úlcera varicosa e tempo de cicatrização com Bota de Unna $(r=60,08 \%$, $\mathrm{p}$-valor $<0,0001, \mathrm{n}=80$ ). Curitiba, PR, Brasil, 2014 
Entre os pacientes em tratamento de úlceras varicosas pesquisados, prevaleceu o sexo masculino, dado que se assemelha ao encontrado em estudo internacional que objetivou identificar as características demográficas e clínicas associadas à diminuição da dor e qualidade de vida de pacientes com úlceras varicosas ativas ${ }^{(9)}$, bem como em outro estudo que avaliou a qualidade de vida em pacientes com úlcera varicosa crônica(10).

Devido ao avanço da idade, as pessoas tornam-se suscetíveis às feridas crônicas, em razão das alterações dos sistemas fisiológicos, modificações nutricionais, metabólicas, vasculares e imunológicas que afetam a função e o aspecto da pele ${ }^{(11)}$. Pessoas idosas, ao serem acometidas por úlceras varicosas, diminuem fisiologicamente a intensidade e velocidade em quase todas as fases de cicatrização, resultando em respostas inflamatórias inferiores, redução de circulação, aumento da fragilidade capilar e do tempo de cicatrização(12). A idade média encontrada nesta pesquisa foi de 57 anos, semelhantes aos achados de outros estudos ${ }^{(13-14)}$.

Nesta pesquisa, o ensino fundamental prevaleceu entre os portadores de úlceras varicosas. Segundo afirmação feita em estudo nacional(15), este perfil de baixa escolaridade é esperado em pacientes que buscam assistência em um ambulatório de serviço público de saúde, bem como o baixo poder aquisitivo.

Frequentemente, os pacientes acometidos pela úlcera varicosa apresentam outras patologias associadas, além da insuficiência venosa crônica, que podem interferir no reparo tecidual. Assim, como em outros estudos ${ }^{(9,14,16)}$, observou-se nos pacientes estudados a predominância de hipertensão arterial sistêmica. Estudo cita $81,5 \%$ de história de diabetes em pacientes acometidos por úlcera varicosa ${ }^{(14)}$, dado divergente ao encontrado nessa pesquisa. Outros autores ${ }^{(17)}$ relatam ausência de outras doenças crônicas associadas em 35\% dos pacientes; sugerem a probabilidade do reparo tecidual em menor tempo, quando comparado a pacientes com várias patologias associadas à insuficiência venosa crônica. Nesta pesquisa notou-se que a maioria dos pacientes com úlcera venosa negou o tabagismo, semelhante a estudo que mostrou apenas $13,3 \%{ }^{(9)}$ e em $24,6 \%^{(14)}$ de pacientes fumantes.

As úlceras varicosas desencadeiam profundas alterações nas atividades de vida diária dos pacientes como, por exemplo, a limitação na mobilidade ${ }^{(17)}$. Em oposição a outro estudo ${ }^{(17)}$, observou-se nesta pesquisa a deambulação sem auxílio na maioria dos pacientes $(89,8 \%)$ e, a análise da variável deambulação mostrou tendência estatística quando comparada ao tempo de cicatrização em até 12 semanas ( $p$-valor $=0,0986$ ), sugerindo que o fato dos pacientes deambularem sem dificuldade influencia negativamente na cicatrização.

Em relação à avaliação do leito da úlcera varicosa, as características predominantes foram presença de esfacelos, exsudato de aspecto seroso, volume moderado e odor discreto. A presença de tecido desvitalizado favorece a infecção ao fornecer nutrientes para o crescimento bacteriano, além de inibir a fagocitose, retardando o reparo tecidual ${ }^{(18)}$.

A presença de exsudato no leito da ferida é um processo fisiológico comum na fase inflamatória da cicatrização, e a avaliação do volume, odor e cor considera a presença ou ausência de infecção ${ }^{(19)}$. A exalação de odor fétido nas úlceras é situação desagradável ao paciente e interfere nas relações pessoais e laborais ${ }^{(20)}$. Registrou-se maioridade de odor discreto nas úlceras varicosas avaliadas nesta pesquisa, dado semelhante ao descrito em estudo realizado em Ambulatório de Reparo de Feridas da região sudeste do Brasil, indicando que $91 \%$ dos pacientes apresentavam odor discreto ${ }^{(21)}$.

Os resultados mostraram tendência para aspecto purulento ( $p$-valor=0,0629) e significância estatística para volume intenso ( $p$-valor=0,0193) do exsudato, variáveis que dificultaram a cicatrização em até doze semanas. Uma ferida exsudativa em excesso pode representar uma fase inflamatória prolongada ou presença de infecção, interferindo no processo de cicatrização ${ }^{(21)}$.

A dor e o desconforto influenciam na adesão ao tratamento e interferem na qualidade de vida do paciente com úlcera varicosa ${ }^{(17)}$. A dor pode ocasionar dificuldade de locomoção, mudança de humor, alterações no relacionamento familiar e convívio social ${ }^{(15)}$. Nesta pesquisa os pacientes referiram dor moderada e intensa, dados que corroboram com a literatura. Estudo registra a dor como característica 
presente na vida do paciente com úlcera varicosa ${ }^{(17)}$.

Os guidelines ${ }^{(22)}$ de tratamento de úlcera varicosa recomendam o uso de cobertura tópica associada à terapia de compressão. Existem várias coberturas e a avaliação do aspecto da ferida, tamanho, tipo de tecido e exsudato, devem ser considerados para a escolha da mais adequada. Há também recomendação de coberturas com prata na presença de infecção, nas feridas criticamente colonizadas ou de cicatrização retardada ${ }^{(23)}$. Cerca de $60 \%$ das úlceras varicosas são colonizadas por bactérias anaeróbias e os curativos com prata são indicados para reduzir a colonização crítica e recomendados para cicatrização de úlceras varicosas, sempre associados a uma terapia compressiva ${ }^{(24)}$.

Nesta pesquisa as coberturas foram utilizadas para a diminuição da infecção no leito da ferida, e as mais empregadas foram: prata nanocristalina 37,5\% ( $n=30)$, curativo de carvão ativado com prata 32,5\% $(\mathrm{n}=26)$. Foram utilizados também coberturas para a diminuição da ferida como curativo hidrocelular $15 \%(n=12)$, hidrofibra com prata $7,5 \%(n=6)$, espuma de silicone suave $5 \%(n=4)$ e curativo de colágeno $1,2 \%(n=2)$.

Salienta-se ainda que 51,3\% dos pacientes estudados tiveram cicatrização das lesões mediante o uso da tecnologia Bota de Unna em até 12 semanas e os demais a cicatrização se deu ao final de um ano. Estudo mostrou que a cicatrização de $40 \%$ das úlceras varicosas com bota de Unna ocorreu no período de um ano ou mais ${ }^{(17)}$. Outros estudos internacionais apontam $40 \%$ a $95 \%$ de cicatrização ${ }^{(25)} \mathrm{e}$ $74,5 \%$ de cicatrização com Bota de Unna ${ }^{(24)}$, mas não há referência sobre o tempo de cicatrização.

Observou-se a predominância de úlceras varicosas de tamanho pequeno, após a mensuração da totalidade das úlceras varicosas nos pacientes pesquisados. Estudo observacional, realizado na região sudeste do Brasil, que tinha como objetivo avaliar as características das úlceras varicosas e citar os produtos indicados para o tratamento, identificou que $22 \%$ das úlceras mediam de $0,1 \mathrm{~cm} 2$ a $10 \mathrm{~cm} 2,8 \%$ mais de $100 \mathrm{~cm} 2$ podendo chegar até $300 \mathrm{~cm} 2$, porém $48 \%$ das úlceras não foram mensuradas ${ }^{(21)}$. Notouse a interferência do tamanho da úlcera varicosa na cicatrização, com significância estatística ( $p$-valor $<0,0001)$. Quanto maior a área da ferida, maior o tempo de cicatrização.

Houve significância estatística ( $p$-valor $<0,0001$ ) na redução da área das úlceras varicosas em 12 semanas, evidenciando que a tecnologia Bota de Unna foi eficaz no tratamento. Percebe-se que a precocidade do início do tratamento da úlcera varicosa de área pequena, com a tecnologia Bota de Unna, aumenta a chance de cicatrização em até 12 semanas.

\section{CONCLUSÃO}

A Bota de Unna foi eficiente na cicatrização das úlceras varicosas em até 12 semanas, em especial nas úlceras de pequeno tamanho. O tamanho da úlcera, a presença de exsudato purulento, volume intenso do exsudato e deambulação sem auxílio são variáveis que interferem na cicatrização. A aplicação da Bota de Unna após a cicatrização pelo período de 3 a 4 semanas foi benéfica para prevenção de recidivas.

O processo de enfermagem aplicado como tecnologia permite aos enfermeiros a melhoria do cuidado prestado e facilita a tomada de decisão mediante o tratamento da úlcera varicosa, pois se baseia em evidências científicas, protocolo institucional e guidelines internacionais. A limitação dessa pesquisa foi a impossibilidade de acesso aos prontuários inativos, ou seja, aqueles que os pacientes foram a óbito ou não realizaram consultas nos últimos dois anos.

Destaca-se ainda que este estudo não é conclusivo, há necessidade de ampliação das investigações em outras instituições, uma vez que não há estudos semelhantes no Brasil para a comparação de dados, no que se refere à cicatrização de úlceras varicosas e recidivas.

Sugere-se a realização de ensaios clínicos, randomizados, com grupo controle nas temáticas sobre tecnologias para prevenção e tratamento de feridas, bem como estudos custo-efetividade.

\section{REFERÊNCIAS}


1. Herranz MT, Corral JCL, Pérez CF. Prevalence of lower extremity vascular ulcers. Systematic review: therapeutic guide design based in pathogenetic and clinicopathological criteria. Reduca. [Internet] 2011; 3(2) [acesso em 23 mar 2016]. Disponível: http://www.revistareduca.es/index.php/reduca-enfermeria/article/viewFile/731/745.

2. Dantas DV, Dantas RAN, Costa IKF, Torres GV. Assistance protocol for venous ulcer patients:validation of contents. Rev Rene. [Internet] 2013; 14(3) [acesso em 23 mar 2016]. Disponível:

http://www.revistarene.ufc.br/revista/index.php/revista/article/view/1204/pdf.

3. do Reis DB, Peres GA, Zuffi FB, Ferreira LA, Poggetto MTD. Care for people with venous ulcers: the perception of nurses in the family health strategy. Reme, Rev. Min. Enferm. [Internet] 2013; 17(1) [acesso em 13 jun 2014]. Disponível: www.reme.org.br/exportar-pdf/582/en_v17n1a09.pdf

4. Yamada BFA. Venous ulcers. In: Jorge SA, Dantas SRPE. Multidisciplinary approuch to wound care. $2^{a}$ ed. São Paulo: Atheneu; 2008.

5. de Macedo EAB, de Oliveira AKA, Torres SMSGSO, Torres GV. Effectiveness of the use of unna boot in the treatment of venous ulcers: a literature review. FIEP Bulletin. [Internet] 2010; 80(n.esp) [acesso em 01 ago 2015]. Disponível: http://www.fiepbulletin.net/index.php/fiepbulletin/article/view/1646/3205.

6. Oliveira AS, Matos JC. Venous ulcer of the lower limbs. Uningá Review. 2010; 4(4): 57-67.

7. Ministério da Saúde (BR). Secretaria de Ciência, Tecnologia e Insumos Estratégicos. Departamento de Ciência e Tecnologia. National policy on health technolgy management. Brasília: Ministério da Saúde; 2009 [acesso em 23 mar 2016]. Disponível:

http://bvsms.saude.gov.br/bvs/publicacoes/methodological_guidelines_technology_assessment_appraisals.pdf.

8. Ministério da Saúde (BR). Conselho Nacional de Saúde. Diretrizes e normas regulamentadoras de pesquisa envolvendo seres humanos. Resolução n. 466, de 12 de dezembro de 2012. Brasília; 2012.

9. Hopman WM, Buchanan M, VanDenKerkhof EG, Harrison MG. Pain and health-related quality of life in people with chronic leg ulcers. Chronic Dis Inj Can. [Internet] 2013; 33(3) [acesso em 24 ago 2014]. Disponível: http:// www.phac-aspc.gc.ca/publicat/hpcdp-pspmc/33-3/ar-07-eng.php.

10. Saraiva DMRF, Bandarra AJF, Agostinho ES, Pereira NMM, Lopes TS. Quality of life of service users with chronic venous ulcers. Rev. Enf. Ref. [Internet] 2013; 3(10) [acesso em 25 jan 2015]. Disponível:

http://dx.doi.org/10.12707/RIII1241.

11. Scemons D, Elston D. Nurse to nurse: cuidados com feridas em enfermagem. $1^{\mathrm{a}}$ ed. Porto Alegre: AMGH; 2011. 12. de Oliveira DC, Gomes AMT, de Pontes PM, da Costa COM. Construção de um paradigma de cuidado e enfermagem pautado nas necessidades humanas e de saúde. Esc. Anna Nery. [Internet] 2011; 15(4) [acesso em 09 jun 2016]. Disponível: http://dx.doi.org/10.1590/S1414-81452011000400025.

13. Green J, Jester R. Health-related quality of life and chronic venous leg ulceration: part 2. Br J Community Nurs. [Internet] 2010; 15(3) [acesso em 23 fev 2014]. Disponível: http://dx.doi.org/10.12968/bjcn.2010.15.Sup1.46906.

14. Torres GV, Costa IKF, Medeiros RKS, de Oliveira AKA, de Souza AJG, Mendes FRP. The characterization of persons with venous ulcer in Brazil and Portugal: comparitive study. Enferm. Glob. [Internet] 2013; 12(32) [acesso em 15 fev 2014]. Disponível:

http://scielo.isciii.es/scielo.php?script=sci_arttext\&pid=S1695-61412013004400005\&lng=es\&nrm=iso\&tlng=pt.

15. Salomé GM, Ferreira LM. Quality of life in patients with venous ulcers treated with Unna's boot compressive therapy. Rev. Bras. Cir. Plást. [Internet] 2012; 27(3) [acesso em 10 mar 2014]. Disponível:

http://dx.doi.org/10.1590/S1983-51752012000300024.

16. da Silva MH, de Jesus MCP, Merighi MAB, de Oliveira DM, Santos SMR, Vicente EJD. Clinical management of venous ulcers in primary health care. Acta paul. enferm. [Internet] 2012; 25(3) [acesso em 25 fev 2014]. Disponível: http://dx.doi.org/10.1590/S0103-21002012000300002.

17. Fonseca $\mathrm{C}$, Franco T, Ramos A, Silva C. The individual with leg ulcer and structured nursing care intervention: a systematic literature review. Rev. esc. enferm. USP. [Internet] 2012; 46(2) [acesso em 14 mai 2014]. Disponível: http://dx.doi.org/10.1590/S0080-62342012000200029. 
18. Mata VE, Porto F, Firmino F. Tempo e custo do procedimento: curativo em úlcera vasculogênica. R. pesq.: cuid. fundam. online. [Internet] 2010; 2(Suppl) [acesso em 08 jun 2016]. Disponível em:

http://dx.doi.org/10.9789/2175-5361.2010.v0i0.\%25p.

19. Paggiaro AO, Teixeira Neto N, Ferreira MC. Treatment of wounds: general principles. Rev Med. [Internet] 2010; 89(3/4) [acesso em 23 abr 2014]. Disponível: http://www.revistas.usp.br/revistadc/article/view/46286/49942.

20. Malagutti W, Kakihara CT. Curativos, estomias e dermatologia: uma abordagem multiprofissional. São Paulo: Martinari; 2010.

21. de Oliveira BGRB, Nogueira GA, de Carvalho MR, de Abreu AM. The characterization of patients with venous ulcer followed at the outpatient wound repair clinic. Rev. Eletr. Enferm. [Internet] 2012; 14(1) [acesso em 08 jul 2014]. Disponível: https://www.fen.ufg.br/fen_revista/v14/n1/pdf/v14n1a18.pdf.

22. Robson MC, Cooper DM, Aslam R, Gould LJ, Harding KG, Margolis DJ, et al. Guidelines for the treatment of venous ulcers. Wound Rep Reg. [Internet] 2006; (14) [acesso em 23 mar 2014]. Disponível:

http://dx.doi.org/10.1111/j.1524-475X.2006.00174.x.

23. Sellmer D, Carvalho CMG, Carvalho DR, Malucelli A. Expert system to support the decision in topical therapy for venous ulcers. Rev. Gaúcha Enferm. [Internet] 2013; 34(2) [acesso em 24 mar 2016]. Disponível:

http://dx.doi.org/10.1590/S1983-14472013000200020.

24. Fradique C, Pupo A, Quaresma L, Palma-Rosa A, Fernandes M, Silva G, et al. Leg ulcer: conservative treatment. Acta Med Port. [Internet] 2011; 24(1) [acesso em 27 jan 2015]. Disponível:

http://www.actamedicaportuguesa.com/revista/index.php/amp/article/view/341/111.

25. Paul JC, Pieper B, Templin TN. Association with chronic venous disease, pain, and quality of life. J Wound Ostomy Continence Nurs. [Internet] 2011; 38(1) [acesso em 05 mar 2014]. Disponível:

https://www.ncbi.nlm.nih.gov/pmc/articles/PMC3086353/. 